\title{
2004 Progress Report for Grant DE-FG03-03NA00076 - Nuclear Level Densities and $\gamma$-ray Strength Functions: Stewardship Science Academic Alliances Program
}

\author{
G. E. Mitchell \\ North Carolina State University, Raleigh, North Carolina, 27695-8202, USA \\ and Triangle Universities Nuclear Laboratory, Durham, North Carolina, 27708-0308, USA
}

\begin{abstract}
To verify the apparent large enhancement of the radiative strength function in light and medium nuclei, the ${ }^{56} \mathrm{Fe}(\mathrm{n}, 2 \gamma)^{57} \mathrm{Fe}$ reaction was measured. The two-step cascade intensities with soft primary transitions confirm the enhancement. The combined results have been published in Physical Review Letters and featured in the Physics News Update. A study of the $\left({ }^{3} \mathrm{He},{ }^{3} \mathrm{He}\right)$ and $\left({ }^{3} \mathrm{He}, \alpha\right)$ reactions performed on ${ }^{117} \mathrm{Sn}$ showed no large enhancement, consistent with results for nuclei in the rare earth region. Data for the $\left({ }^{3} \mathrm{He},{ }^{3} \mathrm{He}\right)$ and $\left({ }^{3} \mathrm{He}, \alpha\right)$ reactions on ${ }^{171,172} \mathrm{Yb}$ have been combined to examine the systematics of level densities and strength functions in ${ }^{170,171,172} \mathrm{Yb}$. Of special interest is the fact that consistent resonance parameters are obtained for the pygmy resonance in ${ }^{171} \mathrm{Yb}$ from the two reactions. A paper on these results has been accepted for publication in Physical Review C. Analysis of data on the ${ }^{48} \mathrm{Ti}(\mathrm{n}, \mathrm{xnypz} \alpha \gamma)$ reaction cross section for $\mathrm{E}_{n}=1$ to $250 \mathrm{MeV}$ obtained with the GEANIE array is now essentially complete. Preliminary measurements of neutron capture on ${ }^{151,153} \mathrm{Eu}$ have been performed with the DANCE array.
\end{abstract}




\section{RESEARCH}

\section{A. Background}

The research program involves a post-doctoral fellow (Dr. Undraa Agvaanluvsan) and a graduate student (Mr. Dugersuren Dashdorj), in addition to the principal investigator. The two younger researchers are stationed at Lawrence Livermore National Laboratory in order to ensure maximum interaction with Livermore personnel. Both moved to Livermore near the end of 2002 and are now well integrated into $\mathrm{N}$ division research activities.

The initial focus of the research proposal was (a) to explore the applicability of a relatively new method (the "Oslo method") to determine simultaneously average level densities and radiative strength functions (a topic of general importance both to stewardship applications and to astrophysics) and (b) to perform measurements of direct relevance for stewardship applications. Dr. Agvaanluvsan first concentrated on the level density-radiative strength function issues with the new method. The availability of the new DANCE array offers excellent opportunities in pure physics and astrophysics, while providing crucial information for stewardship science. This will become the major focus for her. Mr. Dashdorj is actively involved in performing experiments and analyzing data obtained with GEANIE for stewardship science.

\section{B. Level densities and radiative strength functions}

The Oslo method provides average level densities and radiative strength functions. Since information from direct counting methods is used to normalize the experimental level densities and measured neutron capture widths are used to normalize the radiative strength functions, the new information is the energy dependence of the level density $\rho$ above the low-lying levels and below the separation energy, and of the radiative strength function (RSF) at low $\gamma$-ray energies. These properties are extremely difficult to measure at these energies and as a result there is very little direct experimental information available. We 
focus on anomalies in the energy dependence of $\rho$ and RSF.

Since the Oslo method had been shown to work quite well in heavy nuclei, it was important to evaluate the method in lighter nuclei. These nuclei are especially relevant for stewardship science and for astrophysics. Results for both Fe and Mo isotopes were very interesting. The most striking result was the apparent enhancement of the radiative strength function at low energies. This large enhancement is a factor of ten higher than the predictions of standard models for soft $(\leq 2 \mathrm{MeV})$ transitions. This large enhancement was so unexpected that an independent confirmation was needed. We performed a study of the ${ }^{56} \mathrm{Fe}(\mathrm{n}, 2 \gamma){ }^{57} \mathrm{Fe}$ reaction at a research reactor in Budapest, Hungary. Analysis of the two-step cascade intensities confirm the large enhancement of the RSF for soft transitions. These results were recently published in Physical Review Letters.

The large enhancement of the RSF for soft transitions was also observed in several Mo isotopes, but was not observed in numerous studies of rare earth nuclei. We decided to study a nuclide intermediate between these two regions: an experiment on ${ }^{116,117} \mathrm{Sn}$ was performed at the Oslo Cyclotron Laboratory. Preliminary analysis of these data indicate that the large enhancement of the RSF for soft transitions does not occur in Sn.

Prior to the observation of the large enhancement for soft transitions, the most striking feature of the RSF observed with this method was the observation of low energy resonances (pygmy resonances) in all rare earth nuclei measured. We analyzed new data from a ${ }^{171} \mathrm{Yb}$ target and combined these results with earlier data on ${ }^{172} \mathrm{Yb}$ in order to study the systematics of the level densities and radiative strength functions in ${ }^{170,171,172} \mathrm{Yb}$. Results for the RSF from the two reactions $-\left({ }^{3} \mathrm{He},{ }^{3} \mathrm{He}\right)$ and $\left({ }^{3} \mathrm{He}, \alpha\right)$ - lead to consistent parameters characterizing the pygmy resonances. In addition results from the two reactions led to consistent results for the level densities. The fact that the two different reactions give essentially the same results leads to increased confidence in the applicability of statistical $\gamma$-ray spectroscopy. These results have been accepted for publication in Physical Review C.

Future efforts in this area will focus on completing the analysis of the new Sn data (which display statistically significant anomalies in the level density) and of the extensive data set 
for several Mo isotopes (which show large enhancement in the RSF for soft transitions).

\section{Neutron-induced reactions at WNR}

In order to broaden his scientific background, Mr. Dashdorj has participated in a number of experiments performed by the $\mathrm{N}$ division group and presented talks at a number of meetings. In November, 2003, Mr. Dashdorj passed his oral preliminary examination. This examination is the final academic requirement for his Ph.D. degree except for the Ph.D. dissertation.

Mr. Dashdorj's dissertation research is a study of the ${ }^{48} \operatorname{Ti}(\mathrm{n}, \mathrm{xnypz} \alpha \gamma)$ reaction. (The GEANIE array generates a large amount of data for stewardship science. These data are very well suited for dissertation research, since the required experimental and analysis techniques have broad applications in pure and applied nuclear physics.) Cross section measurements were made of prompt $\gamma$-ray production as a function of incident neutron energy on a ${ }^{48} \mathrm{Ti}$ sample enriched to $99.81 \%$; a total of about $4.6 \times 10^{8}$ single- and higher-fold events were acquired. In order to clarify normalization issues, another run was performed with a combined titanium and iron target. Partial $\gamma$-ray cross section were determined for transitions in ${ }^{45-48} \mathrm{Ti},{ }^{44-48} \mathrm{Sc},{ }^{42-45} \mathrm{Ca},{ }^{41-44} \mathrm{~K}$, and ${ }^{41-42} \mathrm{Ar}$. Data were obtained for neutron energies between 1 and $250 \mathrm{MeV}$. The results are compared with model calculations that include compound nuclear and pre-equilibrium emission. The overall result is that calculations using the code EMPIRE predict the general shape of the excitation functions of the reaction $\gamma$-rays. However, the calculations underpredict the measured values in the energy regions where the pre-equilibrium reaction becomes more important.

Pre-equilibrium effects are important when the incident neutron energies are above about $10 \mathrm{MeV}$, but the calculations and experiment do not agree well. We plan to measure the spin distribution transferred in pre-equilibrium neutron induced reactions on targets of ${ }^{170} \mathrm{Er}$ and ${ }^{180} \mathrm{~W}$. The spin distributions will be determined by comparing the relative population of low-lying states with different spins (measured with the GEANIE array) to predictions 
from state of the art pre-equilibrium and Hauser-Feshbach codes. The transferred spin distribution is not well understood nor well characterized in the model calculations.

\section{Neutron capture reactions with DANCE}

The new DANCE array at Los Alamos National Laboratory provides a unique capability for the study of neutron capture reactions. The large efficiency means that very small samples can be studied, even radioactive targets. Measurements in the resonance region should provide key information concerning statistical $\gamma$-ray decay, measurements at slightly higher energies (tens of $\mathrm{keV}$ ) are crucial for astrophysical purposes, while measurements in the 0.1 to $1 \mathrm{MeV}$ region are important for stewardship science.

Preliminary runs have been performed on ${ }^{151} \mathrm{Eu}$ and ${ }^{153} \mathrm{Eu}$. The europium isotopes are of major interest both for astrophysics and for stewardship science. These initial data were obtained during shake-down runs and are not of the quality that is anticipated from the fully operational DANCE facility. We plan to repeat the runs on these two stable europium isotopes. The major development for the next year is the planned measurement of the radioactive ${ }^{155} \mathrm{Eu}$. This target is being prepared by D. Hoffman (LBL) and her group. Her efforts are supported by a separate Academic Alliances grant. Our efforts will focus on analysis of the capture data. This experiment illustrates the interconnectedness of the activity - collaborators include scientists from two LLNL divisions and LBL, as well as members of the local DANCE collaboration at LANL.

We are proposing the study of a number of other targets that are primarily of direct interest for stewardship science, but uncertainties concerning the run cycles at LANL as well as uncertainties related to the processing of data from DANCE make detailed projections

problematic. Our initial focus will be on the europium isotopes. Efforts at DANCE will be central in our ongoing research activities. 


\section{PUBLICATIONS}

\section{Our Recent Relevant Publications - Research initiated prior to start of this}

grant:

1. Level Densities and Radiative Strength Functions in ${ }^{56} \mathrm{Fe}$ and ${ }^{57} \mathrm{Fe}, \mathrm{E}$. Tavukcu, Ph. D. dissertation, North Carolina State University (2002).

2. Thermodynamic Properties of ${ }^{56} \mathrm{Fe}$, E. Tavukcu, J.A. Becker, L.A. Bernstein, M. Guttormsen, E. Melby, G.E. Mitchell, J. Rekstad, A. Schiller, and S. Siem, Frontiers of Nuclear Physics, eds. P. Fallon and R. Clark (AIP Conference Proceedings 656, Melville, New York, 2003), p. 136.

3. Radiative Strength Functions and Level Densities, A. Schiller, J.A. Becker, L.A. Bernstein, A. Voinov, M. Guttormsen, M. Hjorth-Jensen, J. Rekstad, S. Siem, G.E. Mitchell and E. Tavukcu, 11th International Symposium on Capture Gamma-Ray Spectroscopy and Related Topics, eds. J. Kvasil, P. Cejnar, and M. Krticka (World Scientific, Singapore, 2003), p. 432.

4. Level Densities in ${ }^{56,57} \mathrm{Fe}$ and ${ }^{96,97} \mathrm{Mo}$, A. Schiller, E. Tavukcu, L.A. Bernstein, P.E. Garrett, M. Guttormsen, M. Hjorth-Jensen, C.W. Johnson, G.E. Mitchell, J. Rekstad, S. Siem, A. Voinov, and W. Younes, Phys. Rev. C 68, 054326 (2003).

5. Thermodynamic Properties of ${ }^{56} \mathrm{Fe}$, E. Tavukcu, J.A. Becker, L.A. Bernstein, M. Guttormsen, E. Melby, G.E. Mitchell, J. Rekstad, A. Schiller, and S. Siem, Frontiers of Nuclear Physics, eds. P. Fallon and R. Clark (AIP Conference Proceedings 656, Melville, New York, 2003), p. 136.

6. Average Nuclear Level Densities and Radiative Strength Functions in ${ }^{56,57}$ Fe from Primary $\gamma$-ray Spectra, E. Tavukcu, J.A. Becker, L.A. Bernstein, P.E. Garrett, M. Guttormsen, G.E. Mitchell, J. Rekstad, A. Schiller, S. Siem, A. Voinov, and W. Younes, 
Application of Accelerators in Research and Industry: Seventeenth International Conference, eds. J.L. Duggan and I.L. Morgan (AIP Conference Proceedings 680, Melville, New York, 2003), p. 296.

\section{Supported by NNSA:}

\section{PAPERS}

1. Level Densities and Radiative Strength Functions in ${ }^{171} \mathrm{Yb}$ and ${ }^{170} \mathrm{Yb}, \mathrm{U}$. Agvaanluvsan, M. Guttormsen, G.E. Mitchell, J. Rekstad, A. Schiller, and S. Siem, XI International Seminar on Interactions of Neutrons with Nuclei, ISINN-XI (Joint Institute of Nuclear Research, Dubna, Russia, 2004), p. 65.

2. Missing and Spurious Levels in Nuclear Resonances, G.E. Mitchell, S.J. Lokitz, U. Agvaanluvsan, M.P. Pato, and J.F. Shriner, Jr., to be published in the proceedings of XII International Seminar on Interactions of Neutrons with Nuclei, Dubna, Russia.

3. Large enhancement of radiative strength for soft transitions in the quasicontinuum, A. Voinov, E. Algin, U. Agvaanluvsan, T. Belgya, R. Chankova, M. Guttormsen, G.E. Mitchell, J. Rekstad, A. Schiller, and S. Siem, Phys. Rev. Lett. 93, 142504 (2004).

4. Improved Level Densities by Identification of Spurious Levels, U. Agvaanluvsan, G.E. Mitchell, D. Dashdorj, C.M. Frankle, S.F. Lokitz, and J.F. Shriner, Jr., to be published in the proceedings of the International Conference on Nuclear Data, Santa Fe, NM.

5. ${ }^{48} \operatorname{Ti}(\mathrm{n}, \mathrm{xnypz} \alpha \gamma)$ reaction cross section for neutron energies up to $250 \mathrm{MeV}$, D. Dashdorj, P.E. Garrett, J.A. Becker, L.A. Bernstein, J.R. Cooper, M. Devlin, N. Fotiades, G.E. Mitchell, R.O. Nelson, W. Younes, to be published in the proceedings of the International Conference on Nuclear Data, Santa Fe, NM.

6. U. Agvaanluvsan, Investigation of the Radiative Strength Function, to be published in the proceedings of the International Conference on Application of Accelerators, Fort Worth, TX (2004). 
7. Level Densities and $\gamma$-ray Strength Functions in ${ }^{170.171,172} \mathrm{Yb}, \mathrm{U}$. Agvaanluvsan, A. Schiller, J.A. Becker, L.A. Bernstein, P.E. Garrett, M. Guttormsen, G.E. Mitchell, J. Rekstad, S. Siem, A. Voinov, and W. Younes, to be published in Phys. Rev. C.

\section{TALKS}

\section{Invited}

1. Level Densities and Radiative Strength Functions in ${ }^{171} \mathrm{Yb}$ and ${ }^{170} \mathrm{Yb}, \mathrm{U}$. Agvaanluvsan, XI International Seminar on Interactions of Neutrons with Nuclei, ISINN-XI, Dubna, Russia (2004)

2. Comparing the total $\gamma$-ray spectrum for ${ }^{116} \mathrm{Sn}$ from the $\left({ }^{3} \mathrm{He}, \alpha\right)$ and $(\mathrm{n}, \gamma)$ reactions, U. Agvaanluvsan, Nuclear Reactions on Unstable Nuclei and the Surrogate Reaction Technique, Pacific Grove, CA, 2004.

3. Resonances and average nuclear properties using DANCE, U. Agvaanluvsan, Workshop on new opportunities and challenges with DANCE, Santa Fe, NM, 2004.

4. Cross Sections, Level Densities and Strength Functions, G.E. Mitchell, 2004 Stewardship Science Academic Alliances Program Symposium, Albuquerque, NM, 2004.

5. Missing and Spurious Levels in Nuclear Resonances, G.E. Mitchell, XII International Seminar on Interactions of Neutrons with Nuclei, ISINN-XII, Dubna, Russia, 2004.

6. U. Agvaanluvsan, Improved Level Densities by Identification of Spurious Levels, International Conference on Nuclear Data, Santa Fe, NM (2004).

7. U. Agvaanluvsan, Investigation of the Radiative Strength Function, International Conference on Application of Accelerators, Fort Worth, TX (2004).

\section{Contributed}


1. ${ }^{48} \mathrm{Ti}(\mathrm{n}, \mathrm{xnyp} \gamma)$ reaction cross section for $\mathrm{E}_{n}=1$ to $250 \mathrm{MeV}$, D. Dashdorj, P. E. Garrett, J. A. Becker, L. A. Bernstein, J. R. Cooper, M. Devlin, N. Fotiades, G. E. Mitchell, R. O. Nelson, and W. Younes, Sixth LANSCE User Meeting, Los Alamos National Laboratory, October, 2003.

2. Level Densities and Radiative Strength Functions in ${ }^{171} \mathrm{Yb}$ and ${ }^{170} \mathrm{Yb}$, U. Agvaanluvsan, M. Guttormsen, G.E. Mitchell, J. Rekstad, A. Schiller, and S. Siem, Sixth LANSCE Users Meeting, Los Alamos, NM (2003).

3. ${ }^{48} \mathrm{Ti}(\mathrm{n}, \mathrm{xnyp} \gamma)$ reaction cross section using spallation neutrons for $\mathrm{E}_{n}=1$ to $250 \mathrm{MeV}$, D. Dashdorj, P.E. Garrett, J.A. Becker, L.A. Bernstein, J.R. Cooper, W. Younes, G.E. Mitchell, M. Devlin, N. Fotiades, and R.O. Nelson, Bull. Am. Phys. Soc. 48, No. 8, $42(2003)$.

4. Level densities and radiative strength functions in ${ }^{116,117} \mathrm{Sn}, \mathrm{U}$. Agvaanluvsan, G.E. Mitchell, R. Chankova, M. Guttormsen, A.C. Sunde, J.A. Becker, L.A. Bernstein, A. Schiller, and A. Voinov, Bull. Am. Phys. Soc. 48, No. 8, 68 (2003).

5. Density of Levels in ${ }^{116} \mathrm{Sn}, \mathrm{U}$. Agvaanluvsan, G.E. Mitchell, R. Chankova, M. Guttormsen, A.C. Sunde, J.A. Becker, L.A. Bernstein, A. Schiller, and A. Voinov, 2004 Stewardship Science Academic Alliances Program Symposium, Albuquerque, NM, 2004.

6. Partial Gamma-ray Cross Section for the ${ }^{48} \mathrm{Ti}+\mathrm{n}$ Reaction, D. Dashdorj, P.E. Garrett, J.A. Becker, L.A. Bernstein, J.R. Cooper, M. Devlin, N. Fotiades, R.D. Hoffman, G.E. Mitchell, R.O. Nelson, and W. Younes, 2004 Stewardship Science Academic Alliances Program Symposium, Albuquerque, NM, 2004.

7. Neutron Capture Experiments on Eu Isotopes, J. Schwantes, R. Sudowe, C.M.Folden, III, H. Nische, U. Agvaanluvsan, G.E. Mitchell, D.C. Hoffman, 2004 Stewardship Science Academic Alliances Program Symposium, Albuquerque, NM, 2004. 
8. Soft Radiative Strength in Warm Nuclei, A. Schiller, A. Voinov, U. Agvaanluvsan, E. Algin, J.A. Becker, T. Belgya, L.A. Bernstein, R. Chankova, P.E. Garrett, M. Guttormsen, G.E. Mitchell, R.O. Nelson, J. Rekstad, and S. Siem, International Nuclear Physics Conference 2004, Gotheburg, Sweden, 2004.

9. Soft Radiative Strength in Warm Nuclei, A. Schiller, A. Voinov, U. Agvaanluvsan, E. Algin, J.A. Becker, T. Belgya, L.A. Bernstein, R. Chankova, P.E. Garrett, M. Guttormsen, G.E. Mitchell, R.O. Nelson, J. Rekstad, and S. Siem, Nuclei at the Limits, Chicago, IL, 2004.

10. ${ }^{48} \operatorname{Ti}(\mathrm{n}, \mathrm{xnypz} \alpha \gamma)$ reaction cross section for neutron energies up to $250 \mathrm{MeV}$, D. Dashdorj, P.E. Garrett, J.A. Becker, L.A. Bernstein, J.R. Cooper, M. Devlin, N. Fotiades, G.E. Mitchell, R.O. Nelson, W. Younes, to be presented at the Chicago Nuclear Physics meeting. 